\title{
STIMULATION OF GLUTEN-FREE SOURDOUGH FERMENTATION
}

\author{
A. Diowksz* and E. Kordialik-Bogacka \\ Institute of Fermentation Technology and Microbiology, Lodz University of Technology, \\ Wolczanska 171/173, 90-924 Lodz. Poland
}

(Received: 4 October 2012; accepted: 3 January 2013)

\begin{abstract}
Implementation of sourdough technology would increase nutritional value and attractiveness of gluten-free bread. However, fermentation process requires stimulation. The course of fermentation of gluten-free sourdoughs prepared from maize and soy flours and maize and potato starches, enriched in glucose and casein hydrolysate or soy sprouted seeds used as fermentation enhancers was evaluated. The clearest effect of the supplementation was observed in the case of sourdough prepared from starches only. The addition of glucose in the amounts as low as $0.5 \%$ has proved to be enough to enhance fermentation effectively. Supplementation of the sourdoughs with peptides was not very effective; however, when used together with glucose, a synergistic effect could be detected. The addition of sprouted seeds allowed to shorten the fermentation process with up to several hours. These studies have demonstrated possibility to simplify and shorten the process of gluten-free sourdough fermentation by modification of mixtures composition.
\end{abstract}

Keywords: gluten-free bread, sourdough, lactic acid fermentation, celiac disease

Celiac disease defined as gluten-sensitive enteropathy is one of the most often occurring forms of food intolerance, touching up to $1 \%$ of the world's population (NIEWINSKI, 2008). The disease is caused by a specific amino acid sequence present in the prolamin fraction of basic cereals' proteins. In oversensitive persons gluten consumption leads to damage to the small intestine mucosa. It strongly interferes with uptake of nutrients from food, as the destruction of epithelial cells reduces the absorptive surface. So far, a strict exclusion of gluten from the diet is the only treatment (LoHI et al., 2007). Avoiding any products containing toxic prolamins could be very difficult, because in most regions of the world wheat or rye flour is a fundamental component of bread being the staple diet.

Total lifelong avoidance of gluten containing products strongly affects the nutritional profile of the diet. Celiacs consume too little fibre and protein, inadequate amounts of vitamins, and insufficient quantities of minerals (NIEWINSKI, 2008). The possibility of introducing sourdough bread to the gluten-free (GF) diet would increase its nutritional value significantly, because of the unique, healthy properties of it (PoutANen et al., 2009). Fermentation with lactic acid bacteria (LAB) strongly elevates bioavailability of micro and macroelements due to phytate degradation (REALE et al., 2007). Besides, the nutritive value of sourdough bread is elevated as the result of increase in B-group vitamin content as well as exogenic amino acids (PoUTANEN et al., 2009). Moreover, biological acidification of GF dough could enhance bread aroma and taste (HANSEN \& SchieberLe, 2005), which is of high physiological importance. It would significantly affect the wellbeing of patients with celiac disease.

\footnotetext{
* To whom correspondence should be addressed. Fax: +48 4263659 76; e-mail: anna.diowksz@p.lodz.pl
} 
Not less important is the prolonged shelf life of sourdough bread. The last years have experienced an increased interest in the use of lactic acid bacteria as a natural way to prevent growth of moulds and fungi in bread (Moore et al., 2008).

So far only few attempts have been made for producing GF sourdoughs, however, the works aimed at producing a sourdough bread that is tolerated by celiac patients are still continued (Moore et al., 2008; Moroni et al., 2009). The use of LAB starter cultures enables to obtain well acidified GF sourdoughs. However, there is still need for elevating the efficacy of the fermentation process.

The aim of the study was to evaluate the course of one stage fermentation of gluten-free sourdoughs enriched in glucose and casein hydrolysate or soy sprouted seeds used as fermentation enhancers.

\section{Materials and methods}

\subsection{Sourdough fermentation}

The composition of GF mixtures was based on maize and soy flours and maize and potato starches. 'Maize mixture' consisted of maize flour and starch and potato starch in ratio 31:38:31. 'Soy mixture' consisted of soy flour and potato starch in ratio 16:84. 'Starch mixture' consisted of maize and potato starches in ratio 53:47.

Sourdoughs were prepared from GF mixtures and tap water to give a dough yield of 185 , that is, $100 \mathrm{~g}$ GF mixture and $85 \mathrm{~g}$ water. The starter culture was composed of LAB of the Lactobacillus species (Lb. plantarum LOCK-0860, Lb. sanfranciscensis DSM-20663, and Lb. brevis DSM-1267) cultivated in MRS medium at $30{ }^{\circ} \mathrm{C}$ for $24 \mathrm{~h}$. Sourdoughs were inoculated with $2 \%$ (wt/wt) of the starter, which corresponded to the initial LAB cell number of $10^{7} \mathrm{CFU} \mathrm{g} \mathrm{g}^{-1}$ (colony forming units per gram). The fermentation was performed at $30{ }^{\circ} \mathrm{C}$ for $24 \mathrm{~h}$. The progress of fermentation was monitored by total titratable acidity (TTA) determined following the standard analytical method. MRS-agar was used for the enumeration of LAB counts. The plates were incubated at $30{ }^{\circ} \mathrm{C}$ for $72 \mathrm{~h}$.

\subsection{Supplements used in the study}

GF mixtures were supplemented with glucose (pharmaceutical glucose, BTL, Poland) added at dosages of $0.1,0.2,0.3,0.4,0.5,1.0,1.5$, and $2.0 \%$; casein hydrolysate (Pepton K, BTL, Poland) $0.5,1.0,1.5$, and $2.0 \%$; or sprouted soy seeds $1,2,3,4$, and $5 \%$.

\subsection{Preparation of sprouted seeds}

Soy seeds (Glycine max) were steeped overnight before germination performed on grooved trays watered twice a day. The process was carried out for 4 days at room temperature in the day-night cycle. The seedlings were dried at $45^{\circ} \mathrm{C}$ and ground.

\subsection{Statistical analysis}

The results represent the means and standard deviations of triplicate determinations. Statistical significance of the data was determined by Student's $t$-test. 


\section{Results and discussion}

The use of LAB starter culture enabled to perform fermentation of the examined mixtures, however with different effectiveness (Fig. 1). The microbiological analysis showed that lactic acid bacteria in soy and maize mixtures reached the same magnitude of $10^{9} \mathrm{CFU} \mathrm{g}^{-1}$, whereas in starch medium cell count was ten times lower. LAB counts clearly mirrored the differences in the availability of nutrients in the mixtures.

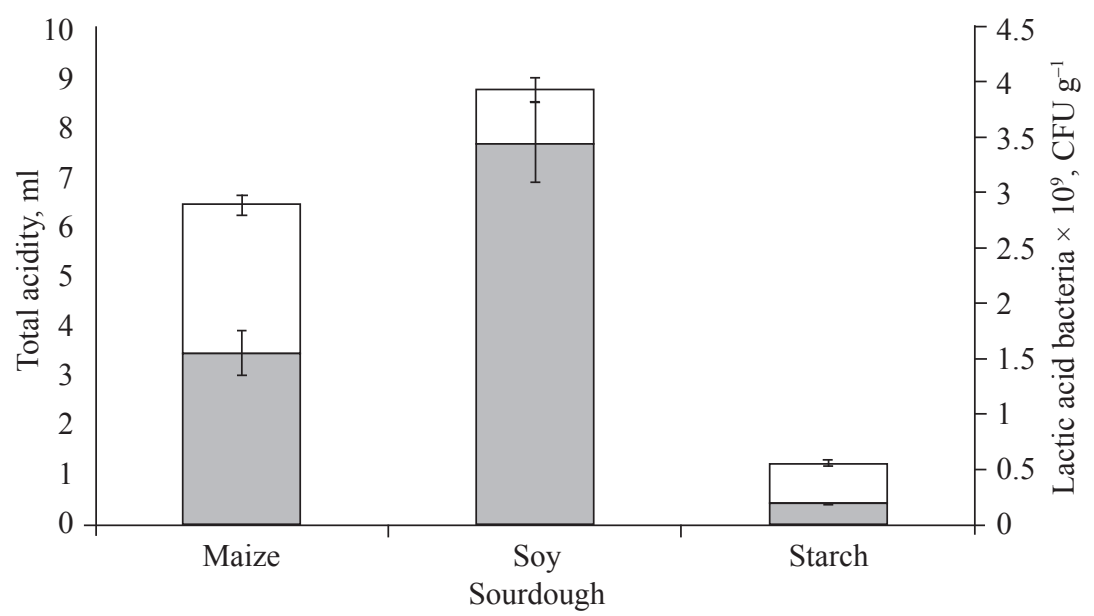

Fig. 1. Total acidity (TTA) of GF sourdoughs and LAB count after $24 \mathrm{~h}$ fermentation at $30^{\circ} \mathrm{C}$. All means within the series are statistically significantly different $(\mathrm{P}<0.01)$. $\square$ : TTA; $\square$ : LAB count

Carbohydrate and nitrogen metabolisms are the key processes to be concerned with (GobBetTi et al., 1994a; 1994b; 2005). Lactic acid bacteria can produce pyruvate and lactate from a number of substrates, including carbohydrates, organic acids, and amino acids. Pyruvate is primarily reduced to lactate, catalysed by lactate dehydrogenase. The main substrates for lactate formation are maltose, glucose, fructose, and malate. The supply of fermentable sugars depends on the balance between starch hydrolysis by the flour and microbial enzymes and microbial consumption (GoвBETTI, 1998; Liv, 2003).

Lactic acid bacteria alone express rather week amylolytic activity (MORONI et al., 2009). The utilization of soluble carbohydrates by LAB and consequently lactic and acetic acid production are greatly influenced by the raw material composition. The examined mixtures contained low amounts of soluble carbohydrates. Soy mixture revealed the highest acidity after the fermentation process mainly due to the nutrients originated from soy flour. As it was expected, starch mixture, poor in fermentable sugars and aminoacids, did not ferment easily. However, to make the process useful for sourdough bread production, additional supplementation of fermentation enhancers was recommended in all cases.

A one-stage fermentation process of GF mixtures with a mixed population of starter culture was used to test an influence of supplements on the fermentation process. The clearest effect of the supplements on the course of fermentation was observed in the case of sourdough prepared from starches only. 
The imbalance between microbial (both LAB and yeast present in sourdough) consumption and starch hydrolysis by flour enzymes leads to the rapid depletion of soluble carbohydrates during sourdough fermentation. This, in turn, decreases LAB acidification due to microbial competition (GoBBETTI, 1998). The addition of selected carbon sources to the dough has been proposed in order to enhance the production of lactic and acetic acids by LAB (CorsetTi et al., 1994). The addition of fructose has been considered by GianotTi and co-workers (1997) as a tool for the enhancement of LAB fermentation. Indeed, the stimulation of bacterial growth in sourdough has only been observed when flour extract supplied with large amounts of the various soluble carbohydrates is used (GoвBEtTI et al., 1994b; GoвветTI, 1998).

In our study, glucose dosage as low as $0.1 \%$ resulted in significant increase $(\mathrm{P}<0.001)$ of the final acidity of starch sourdough, as shown in Fig. 2. However, the number of viable cells of lactic acid bacteria was still very low amounting to $(2.05 \pm 0.21) \cdot 10^{8} \mathrm{CFU} \mathrm{g}^{-1}$. In two other cases, i.e. in maize and soy mixtures, this impact was less pronounced due to the greater flour enzyme activity, which increased the availability of soluble carbohydrates.

The formation of pyruvate and lactate may also derive from the obligatory use of a range of non-conventional substrates, such as amino acids. LAB, therefore, depend on proteolytic systems that allow degradation of proteins (GoBBETTI et al., 2005; Liv, 2003). Thus, addition of protein source could also enhance the fermentation process.

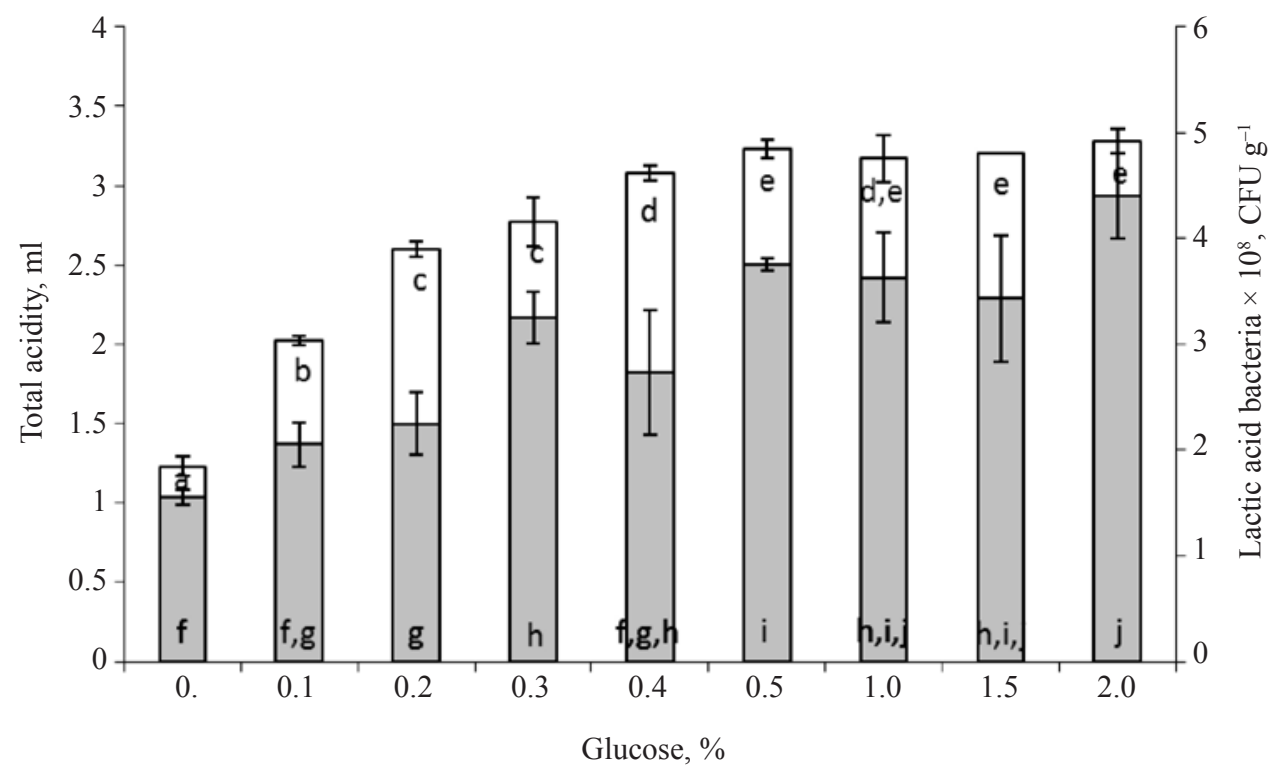

Fig. 2. Total acidity (TTA) of glucose-supplemented starch sourdough and LAB count after $24 \mathrm{~h}$ fermentation at $30^{\circ} \mathrm{C}$. Means signed with different letters $(\mathrm{a}-\mathrm{e} ; \mathrm{f}-\mathrm{j})$ are statistically significantly different $(\mathrm{P}<0.05)$.

$\square:$ TTA; $\square$ : LAB count

The incorporation of dairy ingredients has long been established in the baking industry. They are also used in GF bread for both nutritional and functional benefits (GALLAGHER et al., 2003). However, regardless of the effects on bread quality, the use of milk proteins has some limitations. As a matter of fact, lactose intolerance in celiac patients is a common pathology 
(OJETTI et al., 2005). As it is proposed in the study, the application of casein hydrolysate instead of milk powders may lessen the problem.

Supplementation of GF sourdoughs with peptides was not very effective; however, when used together with glucose, a synergistic effect could be detected, as shown by the example of starch mixture with the poorest nitrogen delivery in Fig. 3.

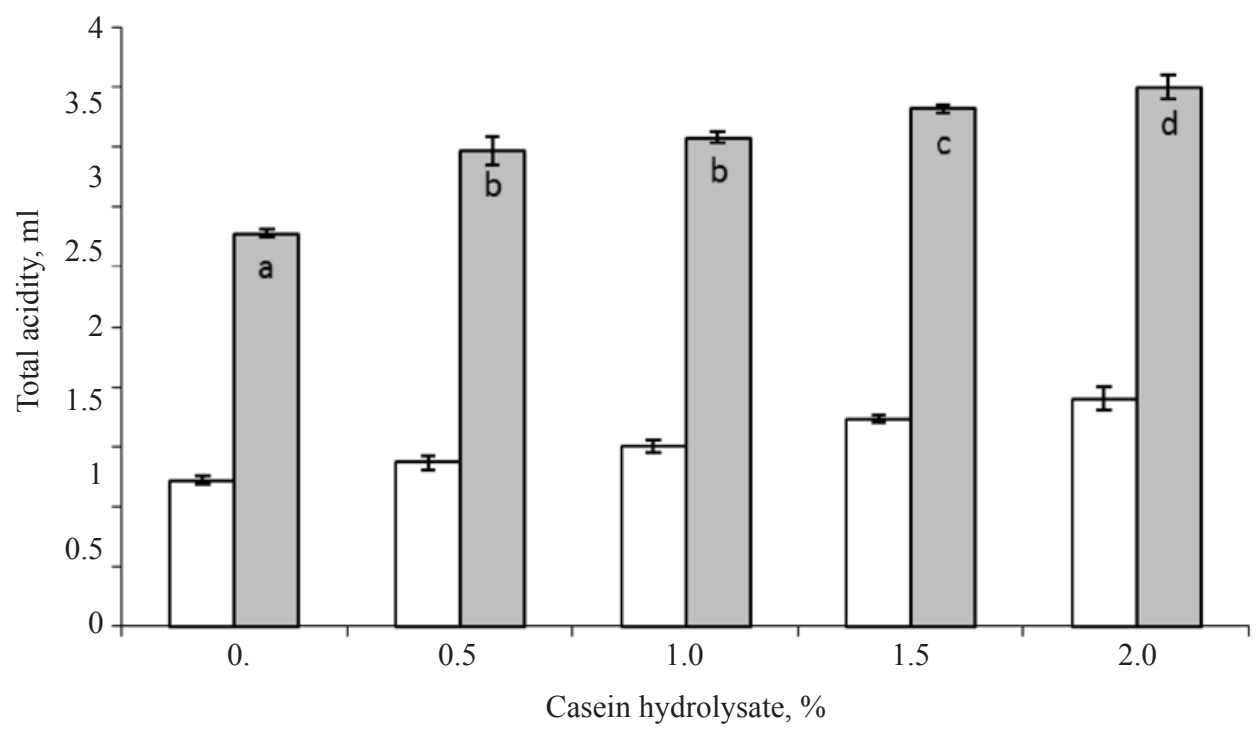

Fig. 3. Total acidity of starch sourdough supplemented with casein hydrolysate and glucose after $24 \mathrm{~h}$ fermentation at $30^{\circ} \mathrm{C}$. All means within the series with no glucose are statistically significantly different $(\mathrm{P}<0.05)$. Means within the series with added glucose, signed with different letters (a-d) are statistically significantly different $(\mathrm{P}<0.05)$.

$\square$ : no glucose; $\square$ : glucose $0.5 \%$

This may result from faster $\mathrm{pH}$ lowering in the presence of glucose, which promotes the breakdown of proteins. The effect of acidification must be considered important for proteolysis in the dough, especially for long sourdough fermentations (THIELE et al., 2002). This enhanced proteolysis is attributed either to the proteolytic activity of sourdough LAB and/or to an activation of proteolysis by cereal enzymes under the acidic conditions of sourdough fermentation (GobBetti et al., 1994a; GobBetti, 1998; Thiele et al., 2002).

Very promising results were obtained with the use of sprouted soy seeds being the source of enzymes and nutrients enhancing LAB growth. In the case of starch mixture, the dosage as low as $1 \%$ of sprouts resulted in raising the number of bacterial cells by one order of magnitude up to the counts of $(1.29 \pm 0.15) \times 10^{9} \mathrm{CFU} \mathrm{\textrm {g } ^ { - 1 }}$. Lactic acid fermentation was noticeably increased and markedly accelerated (Fig. 4). Even the lowest applied dosage of sprouts $(1 \%)$ revealed strong stimulating effect on the rate of acidification resulting in three times higher $(\mathrm{P}<0.001)$ final acidity.

The raw material, very tough for fermentation, after supplementation underwent acidification with dynamics similar to that observed for the maize mixture. The addition of sprouts also enhanced the fermentation of maize sourdough itself, shortening it by $12-16 \mathrm{~h}$, depending on the dose applied (data not shown). 
Intense biochemical processes that occur during sprouting increase the content of free amino acids, sugars, and vitamins as well as active enzymes in grains. The potential of germinated seeds to enable an extensive degradation of proteins in sourdoughs was also indicated by GANZLE and co-workers (2008). However, they suggested the use of plant proteases in wheat or rye doughs to reduce gluten levels to an extent that these products are tolerated by individuals with gluten intolerance.

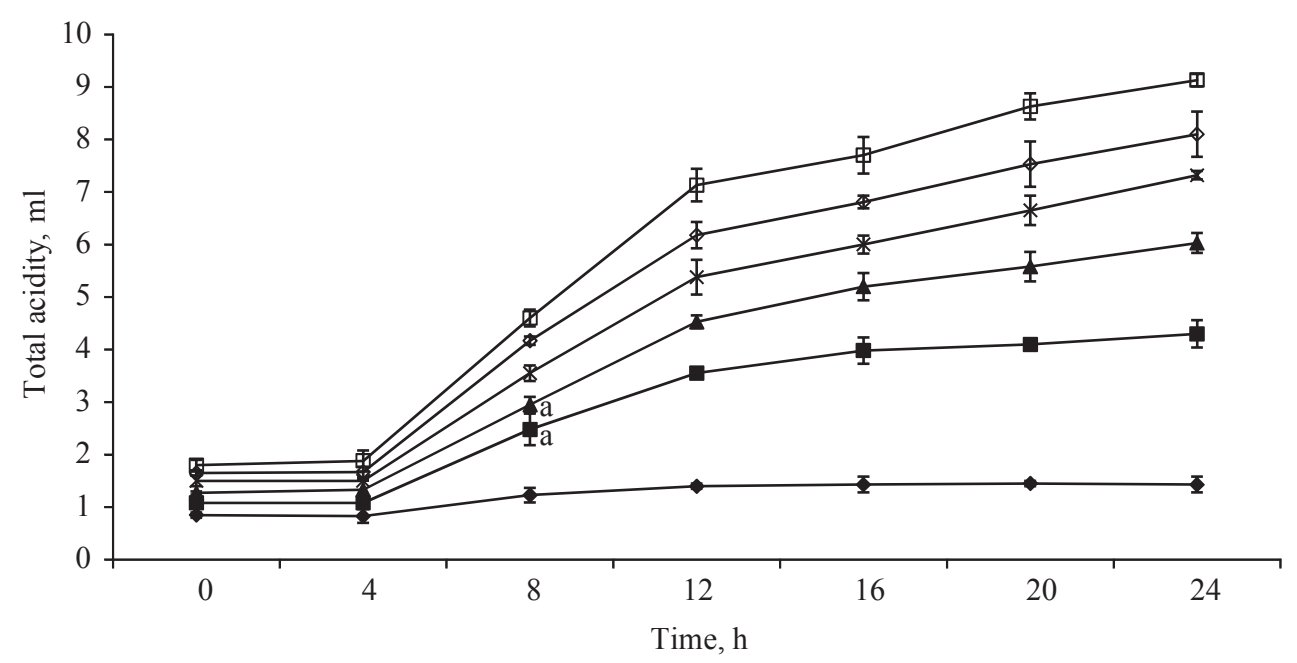

Fig. 4. Progress of fermentation of starch sourdough supplemented with different dosages of soy sprouts. Starting from the $8^{\text {th }}$ hour of fermentation all mean values within a given fermentation hour are statistically significantly different $(\mathrm{P}<0.05)$, with the exception for the values signed with the same (a) letter;

จ: $0 \%, \mathbf{\square}: 1 \% ; \mathbf{\Delta}: 2 \% ; \times: 3 \% ; \diamond: 4 \% ; \square: 5 \%$.

The possibility to implement soy sprouts' biomass into bread recipe was verified in baking trials. As confirmed by a trained sensory panel, the quality of supplemented bread was very satisfying as long as the sprouts' dosage did not exceed $3 \%$.

The results of the study open possibility to widen the assortment of GF breads, which is of enormous importance form the point of view of those touched by celiac disease. The sourdough could be exploited in the production of the range of GF breads designed to satisfy their nutritional needs and reasonably priced. It could meet celiacs' expectations for improving their health and quality of life.

However, it should be mentioned that acceptability of sourdough bread could represent an issue for those consumers who are not used to it. Nevertheless, this problem may be overcome by the selection of appropriate starter cultures and process parameters for obtaining GF sourdough breads with sensorial characteristics that best meet the consumers' requirements (Moroni et al. 2009).

\section{Conclusions}

These studies revealed that it is possible to simplify and shorten the process of GF sourdough fermentation by the modification of mixtures composition, which is very important from the 
point of view of potential application. Sprouted seeds not only enable us to shorten the fermentation process with several hours but may also serve as a source of minerals, vitamins, and dietary fibre elevating the nutritional value of GF bread.

\section{References}

Consetti, A., Gobbetti, M. \& Rossi, J. (1994): Sourdough fermentation in presence of added soluble carbohydrates. Microbiol. Aliments Nutr., 12, 377-385.

Gallagher, E., Gormley, T.R. \& Arendt, E.K. (2003): Crust and crumb characteristics of gluten free breads. $J$. Fd Engng, 56, 153-161.

Ganzle, M.G., Loponen, J. \& Gobbetti, M. (2008): Proteolysis in sourdough fermentations: mechanisms and potential for improved bread quality. Trends Fd Sci. Technol., 19, 513-521.

Gianotti, A., Vannini, L., Gobbetti, M., Corsetti, A., Gardini, F. \& Guerzoni, M. (1997): Modelling of the activity of selected starters during sourdough fermentation. Fd Microbiol., 14, 327-337.

Gobbetti, M. (1998): The sourdough microflora: interactions of lactic acid bacteria and yeasts. Trends Fd Sci. Technol., 9, 267-274.

GobBetti, M., CoRsetti, A. \& Rossi, J. (1994a): The sourdough microflora. Interactions between lactic acid bacteria: metabolism of amino acids. World J. Microbiol. Biotechnol., 10, 275-279.

Gobbetti, M., Corsetti, A. \& Rossi, J. (1994b): The sourdough microflora. Interactions between lactic acid bacteria: metabolism of carbohydrates. Appl. Microbiol. Biotechnol., 41, 456-460.

Gobbetti, M., De Angelis, M., Corsetti, A. \& Di Cagno, R. (2005): Biochemistry and physiology of sourdough lactic acid bacteria. Trends Fd Sci. Technol., 16, 57-69.

Hansen, A. \& Schieberle, P. (2005): Generation of aroma compounds during sourdough fermentation: applied and fundamental aspects. Trends Fd Sci. Technol., 16, 85-94.

Liu, S.Q. (2003): Practical implications of lactate and pyruvate metabolism by lactic acid bacteria in food and beverage fermentations. Int. J. Fd Microbiol., 83, 115-131.

Lohi, S., Mustalahti, K., Kaukinen, K., Laurila, K., Collin, P., Lohi, O., Bravi, E., Gasparin, M., Reunanen, A. \& MAKI, M. (2007): Increasing prevalence of celiac disease over time. Aliment. Pharmacol. Ther, 26, $1217-$ 1225 .

Moore, M.M., Dal Bello, F. \& Arendt, E.K. (2008): Sourdough fermented by Lactobacillus plantarum FST 1.7 improves the quality and shelf life of gluten-free bread. Eur. Fd Res. Technol., 22, 1309-1316.

Moroni, A., Dal Bello, F. \& Arendt, E.K. (2009): Sourdough in gluten-free bread-making: An ancient technology to solve a novel issue? Fd Microbiol., 26, 676-684.

Niewinski, M.M. (2008): Advances in celiac disease and gluten-free diet. J. Am. Diet. Assoc., 108, 661-672.

Ojetti, V., Nucera, G., Migneco, A., Gabrielli, M., Lauritano, C., Danese, S., Zocco, M.A., Nista, E.C., Cammarota, G., De Lorenzo, A., Gasbarrini, G. \& Gasbarrini, A. (2005): High prevalence of celiac disease in patients with lactose intolerance. Digestion, 71, 106-110.

Poutanen, K., Flander, L. \& Katina, K. (2009): Sourdough and cereal fermentation in a nutritional perspective. $F d$ Microbiol., 26, 693-699.

Reale, A., Konietzny, U., Coppola, R., Sorrentino, E. \& Greiner, R. (2007): The importance of lactic acid bacteria for phytate degradation during cereal dough fermentation. J. Agric. Fd Chem., 55, 2993-2997.

Thiele, C., Ganzle, M.G. \& Vogel, R.F. (2002): Contribution of sourdough lactobacilli, yeast and cereal enzymes to the generation of amino acids in dough relevant for bread flavour. Cereal Chem., 79, 45-51. 